\title{
The Large Scale Instability in Rotating Fluid with Small Scale Force
}

\author{
Michael Kopp1, Anatoly Tur², Vladimir Yanovsky¹ \\ ${ }^{1}$ National Academy of Science Ukraine, Institute for Single Crystals, Kharkiv University, Kharkov, Ukraine \\ ${ }^{2}$ Institut de Recherche en Astrophysique et Planétologie, CNRS, Université de Toulouse [UPS], Toulouse, France \\ Email: anatoly.tour@irap.omp.eu
}

Received 14 April 2015; accepted 30 May 2015; published 2 June 2015

Copyright (C) 2015 by authors and Scientific Research Publishing Inc.

This work is licensed under the Creative Commons Attribution International License (CC BY). http://creativecommons.org/licenses/by/4.0/

(c) (7) Open Access

\begin{abstract}
In this paper, we find a new large scale instability in rotating flow forced turbulence. The turbulence is generated by a small scale external force at low Reynolds number. The theory is built on the rigorous asymptotic method of multi-scale development. The nonlinear equations for the instability are obtained at the third order of the perturbation theory. In this article, we explain the nonlinear stage of the instability and the generation vortex kinks.
\end{abstract}

\section{Keywords}

Large Scale Vortex Instability, Coriolis Force, Multi-Scale Development, Small Scale Turbulence, Vortex Kinks

\section{Introduction}

It is well known, that the rotating effects play an important role in many practical and theoretical applications for fluid mechanics [1] and are especially important for geophysics and astrophysics [2] [3] when one has to deal with rotating objects such as Earth, Jupiter and Sun. Rotating fluids could generate different waves and vortex motions, for example, gyroscopic waves, Rossby waves, internal waves, located vortices and coherent vortex structures [4]-[7]. Among the vortex structures, the most interesting are the large scale ones, since they carry out the efficient transport of energy and impulse. The structures which have characteristic scale much more than the scale of turbulence or of the external force which generates this turbulence, are understood as large scale ones. At present, we can state that there are a lot of instabilities which generate the large scale vortex structures (see for example [8]-[14]), in particular, in rotating fluid with the non-homogeneous turbulence [15]. In this work, we find the new large scale instability in rotating fluid, under impact of small external force which keeps up turbulent fluctuations. The nonlinear large scale helical vortex structures of Beltrami type or localized kinks 
with internal helical structure appear as a result of the development of this instability in rotating fluid. We can consider that external small scale force substitutes the action of small scale turbulence. It is supposed that external force is in plane $(X, Y)$, which is perpendicular to the rotation axis, for example, axis $Z$ is directed along the vector of angular velocity of rotation $\boldsymbol{\Omega}$. Helical $2 \mathrm{D}$ field of velocity $W_{x}, W_{y}$ turns around axis $Z$, when $Z$ changes in the kink which links the hyperbolic point and the stable focus (Figure 1). Moreover, this field does some turns in the kink, which links instable and stable focuses (Figure 2). The found instability belongs to the class of instabilities called hydrodynamic $\alpha$-effect. For these instabilities, the positive feedback between velocity components of $W_{x}, W_{y}$ is typical.

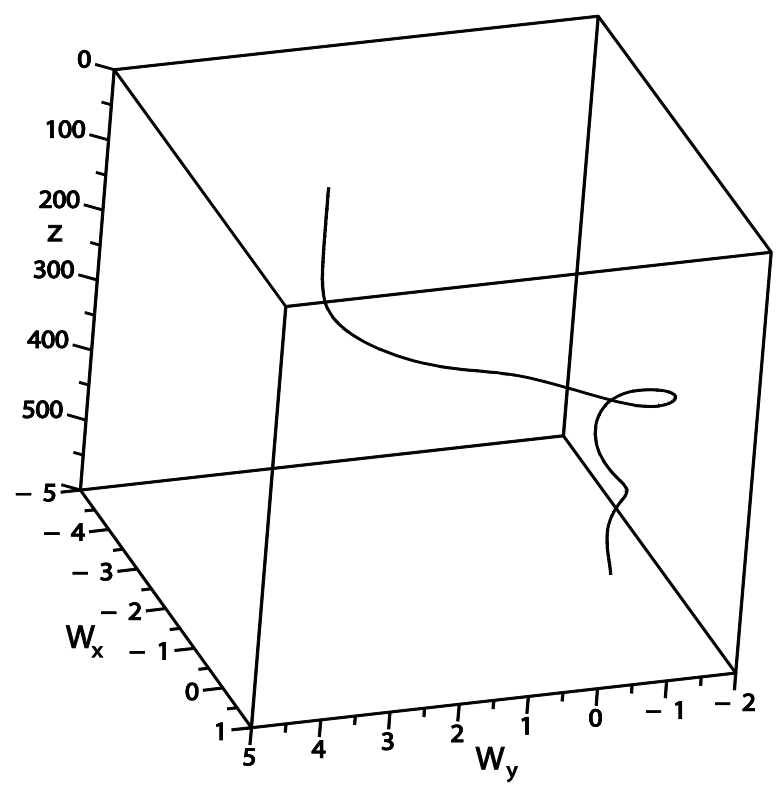

Figure 1. The kink which connects the hyperbolic point with stable knot with $D=1, C_{1}=0.04, C_{2}=0.04$. When approaching the stable knot one can see rotations of velocity field.

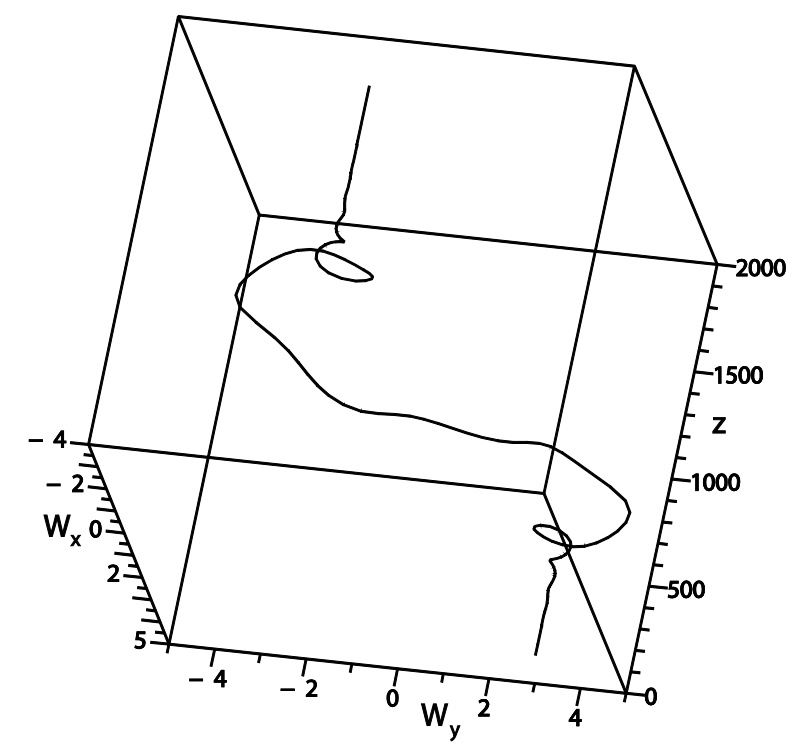

Figure 2. The kink which connects the instable and stable focuses with $D=1, C_{1}=0.04, C_{2}=0.04$. One can see the internal helical structure of the kink. 


$$
\begin{aligned}
& \partial_{T} W_{x}-\Delta W_{x}-\alpha_{y} \frac{\partial}{\partial z} W_{y}=0, \\
& \partial_{T} W_{y}-\Delta W_{y}+\alpha_{x} \frac{\partial}{\partial z} W_{x}=0,
\end{aligned}
$$

and leads to the instability. The $\alpha$-effect is taking its origins from magnetic hydrodynamics, where it engenders the increase of large scale magnetic fields (see for example [16]). It was generalized later for ordinary hydrodynamics. For the time being some examples of hydrodynamics $\alpha$-effect [8]-[14] are already known. From this point of view, in this work we found a new example of $\alpha$-effect. The theory of this instability is developed rigourously using the method of asymptotic multi-scale development, similar to what was done by Frisch, She and Sulem for the theory of the AKA effect [13]. This method allows finding the equations for large scale perturbations as secular equations of asymptotical theory in order to calculate the Reynolds stress tensor and to find the instability. The small parameter of asymptotical development is the Reynolds number $R, R \ll 1$. Our paper is organised as follows: in Section 2 we formulate the problem and the main equations in rotating system of coordinates; in Section 3 we examine the principal scheme of the multi-scale development and we give the secular equations. In Section 4 we calculate the velocity field of zero approximation. In Section 5 we describe the calculations of the Reynolds stress and find the large scale instability. In Section 6 we discuss the saturation of the instability and find non linear stationary vortex structures. The results obtained are discussed in the conclusions given in Section 7.

\section{The Main Equations and Formulation of the Problem}

Let us examine the equations of motion for non-compressible rotating fluid with external force $\boldsymbol{F}_{0}$ in rotating coordinates system:

$$
\begin{aligned}
\frac{\partial \boldsymbol{V}}{\partial t}+(\boldsymbol{V} \nabla) \boldsymbol{V}+2 \boldsymbol{\Omega} \times \boldsymbol{V} & =-\frac{1}{\rho_{0}} \nabla P+v \Delta \boldsymbol{V}+\boldsymbol{F}_{0}, \\
\operatorname{div} \boldsymbol{V} & =0 .
\end{aligned}
$$

The external force $\boldsymbol{F}_{0}$ is divergence-free. Here $\boldsymbol{\Omega}$-angular velocity of fluid rotation, $v$-viscosity, $\rho_{0}$-constant fluid density. Let us design characteristic amplitude of force $f_{0}$, and its characteristic space and time scale $\lambda_{0}$ and $t_{0}$ respectively.

Then $\boldsymbol{F}_{0}=f_{0} \boldsymbol{F}_{0}\left(\frac{\boldsymbol{x}}{\lambda_{0}}, \frac{t}{t_{0}}\right)$. We will design the characteristic amplitude of velocity, generated by external force as $v_{0}$. We choose the dimensionless variables $(t, \boldsymbol{x}, \boldsymbol{V})$ :

$$
\begin{aligned}
& \boldsymbol{x} \rightarrow \frac{\boldsymbol{x}}{\lambda_{0}}, \quad t \rightarrow \frac{t}{t_{0}}, \quad \boldsymbol{V} \rightarrow \frac{\boldsymbol{V}}{v_{0}}, \quad \boldsymbol{F}_{0} \rightarrow \frac{\boldsymbol{F}_{0}}{f_{0}}, \quad P \rightarrow \frac{P}{\rho_{0} P_{0}}, \\
& t_{0}=\frac{\lambda_{0}^{2}}{v}, \quad P_{0}=\frac{v_{0} v}{\lambda_{0}}, \quad f_{0}=\frac{v_{0} v}{\lambda_{0}^{2}}, \quad v_{0}=\frac{f_{0} \lambda_{0}^{2}}{v} .
\end{aligned}
$$

Then, in dimensionless variables the Equation (1) takes forme:

$$
\frac{\partial \boldsymbol{V}}{\partial t}+R(\boldsymbol{V} \cdot \nabla) \boldsymbol{V}+\boldsymbol{D} \times \boldsymbol{V}=-\nabla P+\Delta \boldsymbol{V}+\boldsymbol{F}_{0},
$$

$R=\frac{\lambda_{0} v_{0}}{v},|D|=\sqrt{T a}$ where $R$ and $T a=\frac{4 \Omega^{2} \lambda_{0}^{4}}{v^{2}}$ are respectively the Reynolds number and the Taylor number on scale $\lambda_{0}$. Further we will consider the Reynolds number as small $R \ll 1$ and will construct on this small parameter the asymptitical development. Concerning the parameter $D$, we do not choose any range of values for the moment. Let us examine the following formulation of the problem. We consider the external force as being small scale and of high frequency. This force leads to small scale fluctuations in velocity. After averaging, these quickly oscillating fluctuations vanish. Nevertheless, due to small nonlinear interactions in some orders of perturbation theory, nonzero terms can occur after averaging. This means that they are not oscillatory, that is to 
say, they are large scale. From a formal point of view, these terms are secular, i.e., they create the conditions for the solvability of a large scale asymptotic development. So the purpose of this paper is to find and study the solvability equations, i.e., the equations for large scale perturbations. Let us denote the small scale variables by $x_{0}=\left(x_{0}, t_{0}\right)$, and the large scale ones by $X=(\boldsymbol{X}, T)$. The small scale partial derivative operation $\frac{\partial}{\partial x_{0}^{i}}, \frac{\partial}{\partial t_{0}}$, and the large scale ones $\frac{\partial}{\partial \boldsymbol{X}}, \frac{\partial}{\partial T}$ are written, respectively, as $\partial_{i}, \partial_{t}, \nabla_{i}$ and $\partial_{T}$. To construct a multi-scale asymptotic development we follow the method which is proposed in [16].

\section{The Multi-Scale Asymptotic Development}

Let us search for the solution to Equations (2) and (3) in the following form:

$$
\begin{gathered}
\boldsymbol{V}(\boldsymbol{x}, t)=\frac{1}{R} \boldsymbol{W}_{-1}(X)+\boldsymbol{v}_{0}\left(x_{0}\right)+R \boldsymbol{v}_{1}+R^{2} \boldsymbol{v}_{2}+R^{3} \boldsymbol{v}_{3}+\cdots, \\
T(\boldsymbol{x}, t)=\frac{1}{R} T_{-1}(X)+T_{0}\left(x_{0}\right)+R T_{1}+R^{2} T_{2}+R^{3} T_{3}+\cdots, \\
P(\boldsymbol{x}, t)=\frac{1}{R^{3}} P_{-3}(X)+\frac{1}{R^{2}} P_{-2}(X)+\frac{1}{R} P_{-1}(X)+P_{0}\left(x_{0}\right)+R\left(P_{1}+\bar{P}_{1}(X)\right)+R^{2} P_{2}+R^{3} P_{3}+\cdots
\end{gathered}
$$

Let us introduce the following equalities: $\boldsymbol{X}=R^{2} \boldsymbol{x}_{0}$ and $T=R^{4} t_{0}$ which lead to the expression for the space and time derivatives:

$$
\begin{gathered}
\frac{\partial}{\partial x^{i}}=\partial_{i}+R^{2} \nabla_{i}, \\
\frac{\partial}{\partial t}=\partial_{t}+R^{4} \partial_{T}, \\
\frac{\partial^{2}}{\partial x^{j} \partial x^{j}}=\partial_{j j}+2 R^{2} \partial_{j} \nabla_{j}+R^{4} \partial_{i j} .
\end{gathered}
$$

Using indicial notation, the system of equation can be written as

$$
\begin{gathered}
\left(\partial_{t}+R^{4} \partial_{T}\right) V^{i}+R\left(\partial_{j}+R^{2} \nabla_{j}\right)\left(V^{i} V^{j}\right)+D^{j} \varepsilon_{i j k} V^{k} \\
=\left(\partial_{j}+R^{2} \nabla_{j}\right) P+\left(\partial_{j j}+2 R^{2} \partial_{j} \nabla_{j}+R^{4} \nabla_{j j}\right) V^{i}+F_{0}^{i}, \\
\partial_{t} T-\partial_{j j} T=-V^{z}-R \partial_{j}\left(V^{i} T\right), \\
\left(\partial_{i}+R^{2} \nabla_{i}\right) V^{i}=0 .
\end{gathered}
$$

Substituting these expressions into the initial Equations (2) and (3) and then gathering together the terms of the same order, we obtain the equations of the multi-scale asymptotic development and write down the obtained equations up to order $R^{3}$ inclusive. In the order $R^{-3}$ there is only the equation

$$
\partial_{i} P_{-3}=0 \Rightarrow P_{-3}=P_{-3}(X) \text {. }
$$

In order $R^{-2}$ we have the equation

$$
\partial_{i} P_{-2}=0 \Rightarrow P_{-2}=P_{-2}(X) .
$$

In order $R^{-1}$ we get a system of equations:

$$
\begin{gathered}
\partial_{t} W_{-1}^{i}-\partial_{j j} W_{-1}^{i}+D^{j} \varepsilon_{i j k} W_{-1}^{k}=-\left(\partial_{i} P_{-1}+\nabla_{i} P_{-3}\right)-\partial_{j} W_{-1}^{i} W_{-1}^{j}, \\
\partial_{i} W_{-1}^{i}=0 .
\end{gathered}
$$

The system of Equations (17) and (18) gives the secular terms

$$
-\nabla_{i} P_{-3}=D^{j} \varepsilon_{i j k} W_{-1}^{k},
$$


which corresponds to a geostrophic equilibrum equation.

In zero order $R^{0}$, we have the following system of equations:

$$
\begin{gathered}
\partial_{t} v_{0}^{i}-\partial_{j j} v_{0}^{i}+\partial_{j}\left(W_{-1}^{i} v_{0}^{j}+v_{0}^{i} W_{-1}^{j}\right)+D^{j} \varepsilon_{i j k} v_{0}^{k}=-\left(\partial_{i} P_{0}+\nabla_{i} P_{-2}\right)+F_{0}^{i}, \\
\partial_{i} v_{0}^{i}=0 .
\end{gathered}
$$

These equations give one secular equation:

$$
\nabla P_{-2}=0 \Rightarrow P_{-2}=\text { Const. }
$$

Let us consider the equations of the first approximation $R$ :

$$
\begin{gathered}
\partial_{t} v_{1}^{i}-\partial_{j j} v_{1}^{i}+D^{j} \varepsilon_{i j k} v_{1}^{k}+\partial_{j}\left(W_{-1}^{i} v_{1}^{j}+v_{1}^{i} W_{-1}^{j}+v_{0}^{i} v_{0}^{j}\right)=-\nabla_{j}\left(W_{-1}^{i} W_{-1}^{j}\right)-\left(\partial_{i} P_{1}+\nabla_{i} P_{-1}\right), \\
\partial_{i} V_{1}^{i}+\nabla_{i} W_{-1}^{i}=0 .
\end{gathered}
$$

From this system of equations there follows the secular equations:

$$
\begin{gathered}
\nabla_{i} W_{-1}^{i}=0, \\
\nabla_{j}\left(W_{-1}^{i} W_{-1}^{j}\right)=-\nabla_{i} P_{-1} .
\end{gathered}
$$

The secular Equations (27) and (29) are satisfied by choosing the following geometry for the velocity field (Beltrami field):

$$
\begin{gathered}
W=\left(W_{-1}^{x}(Z), W_{-1}^{y}(Z), 0\right) ; \\
\nabla P_{-1}=0 \Rightarrow P_{-1}=\text { Const. }
\end{gathered}
$$

In the second order $R^{2}$, we obtain the equations

$$
\begin{gathered}
\partial_{t} v_{2}^{i}-\partial_{j j} v_{2}^{i}-2 \partial_{j} \nabla_{j} v_{0}^{i}+\partial_{j}\left(W_{-1}^{i} v_{2}^{j}+v_{2}^{i} W_{-1}^{j}+v_{0}^{i} v_{1}^{j}+v_{1}^{i} v_{0}^{j}\right)+D^{j} \varepsilon_{i j k} v_{2}^{k} \\
=-\nabla_{j}\left(W_{-1}^{i} v_{0}^{j}+v_{0}^{i} W_{-1}^{j}\right)-\left(\partial_{i} P_{2}+\nabla_{i} P_{0}\right), \\
\partial_{i} v_{2}+\nabla_{i} v_{0}=0 .
\end{gathered}
$$

It is easy to see that there are no secular terms in this order.

Let us come now to the most important order $R^{3}$. In this order we obtain the equations

$$
\begin{gathered}
\partial_{t} v_{3}^{i}+\partial_{T} W_{-1}^{i}-\left(\partial_{i j} v_{3}^{i}+2 \partial_{j} \nabla_{j} v_{1}^{i}+\nabla_{i j} W_{-1}^{i}\right)+\nabla_{j}\left(W_{-1}^{i} v_{1}^{j}+v_{1}^{i} W_{-1}^{j}+v_{0}^{i} v_{0}^{j}\right) \\
+\partial_{j}\left(W_{-1}^{i} v_{3}^{j}+v_{3}^{i} W_{-1}^{j}+v_{0}^{i} v_{2}^{j}+v_{2}^{i} v_{0}^{j}+v_{1}^{i} v_{1}^{j}\right)+D^{j} \varepsilon_{i j k} v_{3}^{k}=-\left(\partial_{i} P_{3}+\nabla_{i} \bar{P}_{1}\right), \\
\partial_{i} v_{3}+\nabla_{i} v_{1}=0 .
\end{gathered}
$$

From this we get the main secular equation:

$$
\partial_{T} W_{-1}^{i}-\Delta W_{-1}^{i}+\nabla_{k} \overline{\left(v_{0}^{k} v_{0}^{i}\right)}=-\nabla_{i} \bar{P}_{1} .
$$

There is also an equation to find the pressure $P_{-3}$ :

$$
-\nabla_{i} P_{-3}=D^{j} \varepsilon_{i j k} W_{-1}^{k} .
$$

\section{The Velocity Field in Zero Approximation}

It is clear that the most important is Equation (36). In order to obtain these equations in closed form, we need to calculate the Reynolds stresses $\nabla_{k}\left(\overline{v_{0}^{k} v_{0}^{i}}\right)$. First of all we have to calculate the fields of zero approximation $v_{0}^{k}$. From the asymptotic development in zero order we have

$$
\partial_{t} v_{0}^{i}-\partial_{j j} v_{0}^{i}+W_{-1}^{k} \partial_{k} v_{0}^{i}+D^{j} \varepsilon_{i j k} v_{0}^{k}=-\partial_{i} P_{0}+F_{0}^{i} .
$$


Let us introduce the operator $\hat{D}_{0}$ :

$$
\hat{D}_{0} \equiv \partial_{t}-\partial_{j j}+W^{k} \partial_{k} .
$$

Using $\hat{D}_{0}$, were write Equations (29):

$$
\hat{D}_{0} v_{0}^{i}+D^{j} \varepsilon_{i j k} v_{0}^{k}=-\partial_{i} P_{0}+F_{0}^{i} .
$$

Pressure $P_{0}$ can be found from condition $\operatorname{div} \boldsymbol{V}=0$.

$$
P_{0}=\frac{[\boldsymbol{D} \times \partial]_{i} v_{0}^{i}}{\partial^{2}} .
$$

Let us introduce designations for operatores:

$$
\hat{P}_{i j}=\partial_{j} \frac{[\boldsymbol{D} \times \boldsymbol{\partial}]_{i}}{\partial^{2}}
$$

and for velocities: $v_{0}^{x}=u_{0}, v_{0}^{y}=v_{0}, v_{0}^{z}=w_{0}$. Then excluding pressure from (31), we obtain the system of equations to find the velocity field of zero approximation:

$$
\begin{aligned}
& \left(\widehat{D_{0}}+\widehat{P_{x x}}\right) u_{0}+\left(\widehat{P_{y x}}-D_{z}\right) v_{0}+\left(\widehat{P_{z x}}+D_{y}\right) w_{0}=F_{0}^{x}, \\
& \left(\widehat{P_{x y}}+D_{z}\right) u_{0}+\left(\widehat{D_{0}}+\widehat{P_{y y}}\right) v_{0}+\left(\widehat{P_{z y}}-D_{x}\right) w_{0}=F_{0}^{y}, \\
& \left(\widehat{P_{x z}}-D_{y}\right) u_{0}+\left(\widehat{P_{y z}}+D_{x}\right) v_{0}+\left(\widehat{D_{0}}+\widehat{P_{z z}}\right) w_{0}=F_{0}^{z} .
\end{aligned}
$$

For simplicity, we choose the systeme of coordinates so that the axis $Z$ coincides with the direction of angular velocity of rotation $\Omega$. Then $D_{x}=0, D_{y}=0, D_{z}=D$ In order to solve this system of equations we have to set the force in the explicit form.Let us choose now the external force in the rotating system of coordinates in the following form:

$$
\begin{aligned}
& F_{0}^{z}=0, \boldsymbol{F}_{0 \perp}=f_{0}\left(\boldsymbol{i} \operatorname{Cos} \varphi_{2}+\mathbf{j} \operatorname{Cos} \varphi_{1}\right) ; \varphi_{1}=\boldsymbol{k}_{1} \boldsymbol{x}-\omega_{0} t, \varphi_{2}=\boldsymbol{k}_{2} \boldsymbol{x}-\omega_{0} t, \\
& \boldsymbol{k}_{1}=k_{0}(1,0,1), \boldsymbol{k}_{2}=k_{0}(0,1,1) .
\end{aligned}
$$

It is obvious that divergence of this force us equal to zero. Thus, external force is given in plane $(x, y)$, orthogonal to rotation axis.

The solution for equations system (34) can be found easily in accordance with Cramer's Rule:

$$
u_{0}=\frac{\Delta_{1}}{\Delta}, v_{0}=\frac{\Delta_{2}}{\Delta}, w_{0}=\frac{\Delta_{3}}{\Delta} .
$$

Here $\Delta$ is the determinant of the system (34):

$$
\begin{aligned}
& \Delta=\left|\begin{array}{ccc}
\widehat{D_{0}}+\widehat{P_{x x}} & \widehat{P_{y x}}-D & \widehat{P_{z x}} \\
\widehat{P_{x y}}+D & \widehat{D_{0}}+\widehat{P_{y y}} & \widehat{P_{z y}} \\
\widehat{P_{x z}} & \widehat{P_{y z}} & \widehat{D_{0}}+\widehat{P_{z z}}
\end{array}\right|, \\
& \Delta_{1}=\left|\begin{array}{ccc}
F_{0}^{x} & \widehat{P_{y x}}-D & \widehat{P_{z x}} \\
F_{0}^{y} & \widehat{D_{0}}+\widehat{P_{y y}} & \widehat{P_{z y}} \\
0 & \widehat{P_{y z}} & \widehat{D_{0}}+\widehat{P_{z z}}
\end{array}\right|, \\
& \Delta_{2}=\left|\begin{array}{ccc}
\widehat{D_{0}}+\widehat{P_{x x}} & F_{0}^{x} & \widehat{P_{z x}} \\
\widehat{P_{x y}}+D & F_{0}^{y} & \widehat{P_{z y}} \\
\widehat{P_{x z}} & 0 & \widehat{D_{0}}+\widehat{P_{z z}}
\end{array}\right|,
\end{aligned}
$$




$$
\Delta_{3}=\left|\begin{array}{ccc}
\widehat{D_{0}}+\widehat{P_{x x}} & \widehat{P_{y x}}-D & F_{0}^{x} \\
\widehat{P_{x y}}+D & \widehat{D_{0}}+\widehat{P_{y y}} & F_{0}^{y} \\
\widehat{P_{x z}} & \widehat{P_{y z}} & 0
\end{array}\right| .
$$

After writing down the determinants in the explicit form, we obtain:

$$
\begin{aligned}
u_{0}= & \frac{1}{\Delta}\left[\left(\widehat{D_{0}}+\widehat{P_{y y}}\right)\left(\widehat{D_{0}}+\widehat{P_{z z}}\right)-\left(\widehat{P_{y z}}\right)\left(\widehat{P_{z y}}\right)\right] F_{0}^{x} \\
& +\frac{1}{\Delta}\left[\left(\widehat{P_{z x}}\right)\left(\widehat{P_{y z}}\right)-\left(\widehat{P_{y x}}-D\right)\left(\widehat{D_{0}}+\widehat{P_{z z}}\right)\right] F_{0}^{y}, \\
v_{0}= & \frac{1}{\Delta}\left[\left(\widehat{P_{x z}}\right)\left(\widehat{P_{z y}}\right)-\left(\widehat{P_{x y}}+D\right)\left(\widehat{D_{0}}+\widehat{P_{z z}}\right)\right] F_{0}^{x} \\
& +\frac{1}{\Delta}\left[\left(\widehat{D_{0}}+\widehat{P_{x x}}\right)\left(\widehat{D_{0}}+\widehat{P_{z z}}\right)-\left(\widehat{P_{x z}}\right)\left(\widehat{P_{z x}}\right)\right] F_{0}^{y}, \\
w_{0}= & \frac{1}{\Delta}\left[\left(\widehat{P_{x y}}+D\right)\left(\widehat{P_{y z}}\right)-\left(\widehat{P_{x z}}\right)\left(\widehat{D_{0}}+\widehat{P_{y y}}\right)\right] F_{0}^{x} \\
& +\frac{1}{\Delta}\left[\left(\widehat{P_{x z}}\right)\left(\widehat{P_{y x}}-D\right)-\left(\widehat{D_{0}}+\widehat{P_{x x}}\right)\left(\widehat{P_{y z}}\right)\right] F_{0}^{y} . \\
\Delta= & \left(\widehat{D_{0}}+\widehat{P_{x x}}\right)\left[\left(\widehat{D_{0}}+\widehat{P_{y y}}\right)\left(\widehat{D_{0}}+\widehat{P_{z z}}\right)-\left(\widehat{P_{y z}}\right)\left(\widehat{P_{z y}}\right)\right] \\
& -\left(\widehat{P_{y x}}-D\right)\left[\left(\widehat{P_{x y}}+D\right)\left(\widehat{D_{0}}+\widehat{P_{z z}}\right)-\left(\widehat{P_{x z}}\right)\left(\widehat{P_{z y}}\right)\right] \\
& +\left(\widehat{P_{z x}}\right)\left[\left(\widehat{P_{x y}}+D\right)\left(\widehat{P_{y z}}\right)-\left(\widehat{D_{0}}+\widehat{P_{y y}}\right)\left(\widehat{P_{x z}}\right)\right] .
\end{aligned}
$$

In order to calculate the expressions (40)-(43) we present the external force in complex form:

$$
F_{0}^{x}=\frac{f_{0}}{2}\left(\mathrm{e}^{i \varphi_{2}}+\mathrm{e}^{-i \varphi_{2}}\right), \quad F_{0}^{y}=\frac{f_{0}}{2}\left(\mathrm{e}^{i \varphi_{1}}+\mathrm{e}^{-i \varphi_{1}}\right) .
$$

Then all operators in formulae (40)-(42) act from the left on their eigenfunctions. In particular:

$$
\begin{aligned}
& \widehat{D_{0}} \mathrm{e}^{\mathrm{i} \varphi_{2}}=\mathrm{e}^{\mathrm{i} \varphi_{2}} \widehat{D_{0}}\left(\boldsymbol{k}_{2},-\omega_{0}\right), \widehat{D_{0}} \mathrm{e}^{\mathrm{i} \varphi_{1}}=\mathrm{e}^{\mathrm{i} \varphi_{1}} \widehat{D_{0}}\left(\boldsymbol{k}_{1},-\omega_{0}\right), \\
& \Delta \mathrm{e}^{\mathrm{i} \varphi_{2}}=\mathrm{e}^{\mathrm{i} \varphi_{2}} \Delta\left(\boldsymbol{k}_{2},-\omega_{0}\right), \Delta \mathrm{e}^{i \varphi_{1}}=\mathrm{e}^{\mathrm{i} \varphi_{1}} \Delta\left(\boldsymbol{k}_{1},-\omega_{0}\right) .
\end{aligned}
$$

To simplify the formulae, let us choose $k_{0}=1, \omega_{0}=1$.

We will designate

$$
\widehat{D_{0}}\left(\boldsymbol{k}_{2},-\omega_{0}\right)=2+i\left(w_{y}-1\right)=A_{y}, \widehat{D_{0}}\left(\boldsymbol{k}_{1},-\omega_{0}\right)=2+i\left(w_{x}-1\right)=A_{x} .
$$

Before doing further calculations, we have to note that some components of tensors $\hat{P}_{i j}\left(\boldsymbol{k}_{1}\right)$ and $\hat{P}_{i j}\left(\boldsymbol{k}_{2}\right)$ vanish. Let us write the non-zero components only:

$$
\hat{P}_{y x}\left(\boldsymbol{k}_{1}\right)=\frac{1}{2} D, \hat{P}_{x z}\left(\boldsymbol{k}_{2}\right)=-\frac{1}{2} D, \hat{P}_{x y}\left(\boldsymbol{k}_{2}\right)=-\frac{1}{2} D, \hat{P}_{y z}\left(\boldsymbol{k}_{1}\right)=\frac{1}{2} D .
$$

Taking into account the formulae (45)-(47), we can find the determinant:

$$
\Delta\left(\boldsymbol{k}_{1}\right)=A_{x}^{3}+\frac{1}{2} D^{2} A_{x}, \Delta\left(\boldsymbol{k}_{2}\right)=A_{y}^{3}+\frac{1}{2} D^{2} A_{y} .
$$

In a similar way we find velocity field of zero approximation:

$$
u_{0}=f_{0} \frac{A_{y}}{2} \frac{\mathrm{e}^{i \varphi_{2}}}{A_{y}^{2}+\frac{1}{2} D^{2}}+f_{0} \frac{D}{4} \frac{\mathrm{e}^{i \varphi_{1}}}{A_{x}^{2}+\frac{1}{2} D^{2}}+C . C .,
$$




$$
\begin{aligned}
& v_{0}=-f_{0} \frac{D}{4} \frac{\mathrm{e}^{i \varphi_{2}}}{A_{y}^{2}+\frac{1}{2} D^{2}}+f_{0} \frac{A_{x}}{2} \frac{\mathrm{e}^{i \varphi_{1}}}{A_{x}^{2}+\frac{1}{2} D^{2}}+C . C ., \\
& w_{0}=f_{0} \frac{D}{4} \frac{\mathrm{e}^{i \varphi_{2}}}{A_{y}^{2}+\frac{1}{2} D^{2}}-f_{0} \frac{D}{4} \frac{\mathrm{e}^{i \varphi_{1}}}{A_{x}^{2}+\frac{1}{2} D^{2}}+C . C . .
\end{aligned}
$$

\section{Reynolds Stress and Large Scale Instability}

To close the Equations (27) we have to calculate the Reynolds stresses $\overline{w_{0} u_{0}}$ and $\overline{w_{0} v_{0}}$.

These terms are easily calculated with help of formulae (49)-(51). As a result we obtain:

$$
\begin{aligned}
& \overline{w_{0} u_{0}}=\frac{f_{0}^{2}}{2} \frac{D}{\left|A_{y}^{2}+\frac{1}{2} D^{2}\right|^{2}}-\frac{f_{0}^{2}}{8} \frac{D^{2}}{\left|A_{x}^{2}+\frac{1}{2} D^{2}\right|^{2}}, \\
& \overline{w_{0} v_{0}}=-\frac{f_{0}^{2}}{8} \frac{D^{2}}{\left|A_{y}^{2}+\frac{1}{2} D^{2}\right|^{2}}-\frac{f_{0}^{2}}{2} \frac{D}{\left|A_{x}^{2}+\frac{1}{2} D^{2}\right|^{2}} .
\end{aligned}
$$

Now Equations (27) are closed and take form:

$$
\begin{aligned}
& \partial_{T} W_{x}-\Delta W_{x}+\frac{\partial}{\partial z} \overline{w_{0} u_{0}}=0, \\
& \partial_{T} W_{y}-\Delta W_{y}-\frac{\partial}{\partial z} \overline{w_{0} v_{0}}=0 .
\end{aligned}
$$

We calculate the modules and write the Reynolds stresses (52) in the explicit form:

$$
\begin{aligned}
& \overline{w_{0} u_{0}}=\frac{f_{0}^{2}}{2} \frac{D}{16\left(w_{y}-1\right)^{2}+\left[4+\frac{1}{2} D^{2}-\left(w_{y}-1\right)^{2}\right]^{2}}-\frac{f_{0}^{2}}{8} \frac{D^{2}}{16\left(w_{x}-1\right)^{2}+\left[4+\frac{1}{2} D^{2}-\left(w_{x}-1\right)^{2}\right]^{2}}, \\
& \overline{w_{0} v_{0}}=-\frac{f_{0}^{2}}{8} \frac{D^{2}}{16\left(w_{y}-1\right)^{2}+\left[4+\frac{1}{2} D^{2}-\left(w_{y}-1\right)^{2}\right]^{2}}-\frac{f_{0}^{2}}{2} \frac{D}{16\left(w_{x}-1\right)^{2}+\left[4+\frac{1}{2} D^{2}-\left(w_{x}-1\right)^{2}\right]^{2}} .
\end{aligned}
$$

With small $W_{x}, W_{y}$ Reynolds stresses (52) can be expanded in a series in the small parameters $W_{x}, W_{y}$. Taking into account the formula:

$$
\frac{1}{\left|A_{x, y}^{2}+\frac{1}{2} D^{2}\right|^{2}}=\text { Const. }-\frac{32\left(D^{2}-10\right)}{\left[\left(D^{2}+6\right)^{2}+64\right]^{2}} w_{x, y}+\cdots
$$

We obtain the linearized Equations (53):

$$
\begin{aligned}
& \frac{\partial}{\partial T} W_{x}-\frac{\partial^{2}}{\partial z^{2}} W_{x}-\frac{\alpha f_{0}^{2} D}{2} \frac{\partial}{\partial z} W_{y}+\frac{\alpha f_{0}^{2} D^{2}}{8} \frac{\partial}{\partial z} W_{x}=0, \\
& \frac{\partial}{\partial T} W_{y}-\frac{\partial^{2}}{\partial z^{2}} W_{y}+\frac{\alpha f_{0}^{2} D^{2}}{8} \frac{\partial}{\partial z} W_{y}+\frac{\alpha f_{0}^{2} D}{2} \frac{\partial}{\partial z} W_{x}=0 . \\
& \alpha=\frac{32\left(10-D^{2}\right)}{\left[\left(D^{2}+6\right)^{2}+64\right]^{2}} .
\end{aligned}
$$

We will search for the solution of linear system (55) in the form: 


$$
W_{x}, W_{y} \sim \exp (\gamma T+i k Z) .
$$

We substitute (56) in Equation (55) and obtain the dispersion equation:

$$
\gamma=-i k \frac{\alpha f_{0}^{2} D^{2}}{8} \pm k \frac{\alpha f_{0}^{2} D}{2}-k^{2}
$$

The dispersion Equation (57) shows that equation system (55) has instable oscillatory solutions with oscillatory frequency $\omega=k \frac{\alpha f_{0}^{2} D^{2}}{8}$ and instability growth rate $\gamma=k \frac{\alpha f_{0}^{2} D}{2}-k^{2}$. The instability is large scale because the instable term dominates over dissipation on large scales: $\frac{\alpha f_{0}^{2} D}{2} \succ k$. The maximum growth rate of instability is equal to $\gamma_{\max }=\frac{\alpha^{2} f_{0}^{4} D^{2}}{16}$, and is achieved on the wave vector $k_{\max }=\frac{\alpha f_{0}^{2} D}{4}$. As a result of the development of instability the large scale helical circular polarized vortices of Beltrami type are generated in the system.

\section{Saturation of Instability and Nonlinear Vortex Structures}

It is clear that with increasing of amplitude nonlinear terms decrease and instability becomes saturated. Consequently stationary nonlinear vortex structures are formed. To find these structures let us choose for Equations (54) $\frac{\partial}{\partial T}=0$ and integrate equations one time over $Z$. We obtain the system of equations:

$$
\begin{aligned}
\frac{\mathrm{d}}{\mathrm{d} Z} W_{x} & =\overline{w_{0} u_{0}}+C_{1}, \\
\frac{\mathrm{d}}{\mathrm{d} Z} W_{y} & =\overline{w_{0} v_{0}}+C_{2} .
\end{aligned}
$$

From Equations (58) follows:

$$
\frac{\mathrm{d} w_{x}}{\mathrm{~d} w_{y}}=\frac{\overline{w_{0} u_{0}}+C_{1}}{\overline{w_{0} v_{0}}+C_{2}}
$$

After integrating the system of Equations (59) we obtain:

$$
\int \overline{w_{0} v_{0}} \mathrm{~d} w_{x}+C_{2} w_{x}=\int \overline{w_{0} u_{0}} \mathrm{~d} w_{y}+C_{1} w_{y} .
$$

Integrals in expression (60) are calculated in elementary functions (see [17]), which give the expression for first integral of motion $J$ of Equations (59):

$$
\begin{aligned}
J= & \frac{D^{2}}{8} \frac{w_{x}}{\left[4+\frac{1}{2} D^{2}-\left(w_{y}-1\right)^{2}\right]^{2}+16\left(w_{y}-1\right)^{2}}+\frac{D}{2^{5 / 2}\left(8+D^{2}\right)} \ln \frac{\left(w_{x}-1\right)^{2}+\left(w_{x}-1\right) D \sqrt{2}+4+\frac{1}{2} D^{2}}{\left(w_{x}-1\right)^{2}-\left(w_{x}-1\right) D \sqrt{2}+4+\frac{1}{2} D^{2}} \\
& +\frac{D}{8\left(8+D^{2}\right)} \operatorname{arctg} \frac{\left(w_{x}-1\right)^{2}-4-\frac{1}{2} D^{2}}{4\left(w_{x}-1\right)}-\frac{D^{2}}{8} \frac{w_{y}}{\left[4+\frac{1}{2} D^{2}-\left(w_{x}-1\right)^{2}\right]^{2}+16\left(w_{x}-1\right)^{2}} \\
& +\frac{D}{2^{5 / 2}\left(8+D^{2}\right)} \ln \frac{\left(w_{y}-1\right)^{2}+\left(w_{y}-1\right) D \sqrt{2}+4+\frac{1}{2} D^{2}}{\left(w_{y}-1\right)^{2}-\left(w_{y}-1\right) D \sqrt{2}+4+\frac{1}{2} D^{2}}+\frac{D}{8\left(8+D^{2}\right)} \operatorname{arctg} \frac{\left(w_{y}-1\right)^{2}-4-\frac{1}{2} D^{2}}{4\left(w_{y}-1\right)}+C_{1} w_{y}+C_{2} w_{x} .
\end{aligned}
$$

Equations (58) can be easily calculated numerically using standard tools. In particular, this allows to construct phase portrait of the dynamical system (58) (Figure 3) and to get the most interesting solutions which link singular points on phase plane. See for example Figure 1, where the hyperbolic singular point is connected with 


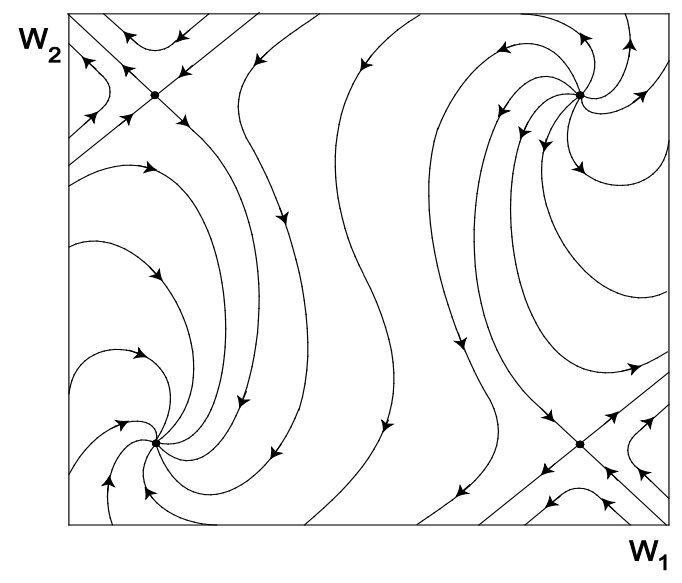

Figure 3. Phase portrait of the dynamical system (58), with $D=1, C_{1}=-0.03, C_{2}=0.03$. One can see two hyperbolic singular points and stable and instable knots.

the stable knot and Figure 2, where the solution connects instable and stable focuses. All these solutions correspond to the large scale localized vortex structures of kink type with rotation, generated by the instability which has been found in this work.

\section{Conclusions and Discussion of the Results}

In this work we find the new large scale instability in rotating fluid. It is supposed that the small scale vortex external force in rotating coordinates system acts on fluid which maintains the small velocity field fluctuations (small scale turbulence with small Reynolds number $R, R \ll 1$ ). For the real applications this Reynolds number should be calculated with help of the turbulent viscosity. The asymptotic development of motion equations by small Reynolds number allows obtaining motion equations for the large scale. These equations are of the hydrodynamic $\alpha$-effect type, in which velocity components $W_{x}, W_{y}$ are connected by the positive feedback. This may result in the appearance of the large scale vortex instability. The large scale vortices of Beltrami type are formed due to this instability in rotating fluid with small scale exterior force. With further increase of amplitude, the instability stabilizes and passes to stationary mode. In this mode the nonlinear stationary vortex structures form. Different vortex kinks belong to the most interesting structures. These kinks link stationary points of dynamical system (58). Kinks which link the hyperbolic point with the stable knot rotate around the stable knot as shown on Figure 1. In the kink which links instable and stable focuses, vector field turns around two singular points, see Figure 2.

Let us note that unlike previous works about hydrodynamic $\alpha$-effect in rotating fluid, the use of the asymptotic development allows constructing naturally the nonlinear theory and studying the stationary nonlinear vortex kinks.

\section{References}

[1] Grinspen, H.P. (1990) The Theory of Rotating Fluids. Breukelen Press, Brookline.

[2] Roberts, P.H. and Soward, A.M. (1978) Rotating Fluids in Geophysics. Academic Press, London.

[3] Clarke, C. and Carswell, B. (2007) Principles of Astrophysical Fluid Dynamics. Cambridge University Press, Cambridge. http://dx.doi.org/10.1017/CBO9780511813450

[4] Vallis, G.K. (2010) Atmospheric and Oceanic Fluid Dynamics. Cambridge University Press, Cambridge.

[5] Abramowicz, M.A., Lanza, A., Spigel, E.A. and Szuszkiewicz, E. (1992) Vortices on Accretion Disks. Nature, 356, 41-43. http://dx.doi.org/10.1038/356041a0

[6] Brandt, P.N., Scharmer, G.B., Ferguson, S., Shine, R.A., Tarbell, T.D. and Title, A.M. (1988) Vortex Flow in the Solar Photosphere. Nature, 335, 238. http://dx.doi.org/10.1038/335238a0

[7] Dritschel, G. and Legras, B. (1993) Modeling Oceanic and Atmospheric Vortices. Physics Today, 46, 44. 
http://dx.doi.org/10.1063/1.881375

[8] Moiseev, S.S., Sagdeev, R.Z., Tur, A.V., Khomenko, G.A. and Yanovsky, V.V. (1983) A Theory of Large-Scale Structure Origination in Hydrodynamic Turbulence. Soviet Physics—JETP, 58, 1149.

[9] Moiseev, S.S., Rutkevich, P.B., Tur, A.V. and Yanovsky, V.V. (1988) Vortex Dynamos in a Helical Turbulent Convection. Soviet Physics-JETP, 67, 294.

[10] Lupyan, E.A., Mazurov, A.A., Rutkevich, P.B. and Tur, A.V. (1992) Generation of Large-Scale Vortices through the Action of Spiral Turbulence of a Convective Nature. Soviet Physics-JETP, 75, 833.

[11] Khomenko, G.A., Moiseev, S.S. and Tur, A.V. (1991) The Hydrodynamic Alpha-Effect in a Compressible Fluid. Journal of Fluid Mechanics, 225, 355. http://dx.doi.org/10.1017/S0022112091002082

[12] Levina, G.V., Moiseev, S.S. and Rutkevich, P.B. (2000) Hydrodynamic Alpha-Effect in a Convective System. Advances in Fluid Mechanics, 25, 111.

[13] Frisch, U., She, Z.S. and Sulem, P.L. (1987) Large-Scale Flow Driven by the Anisotropic Kinetic Alpha Effect. Physica D, 28, 382. http://dx.doi.org/10.1016/0167-2789(87)90026-1

[14] Tur, A.V. and Yanovsky, V.V. (2013) Non Linear Vortex Structures in Stratified Fluid Driven by Small-Scale Helical Force. Open Journal of Fluid Dynamics, 3, 64. http://dx.doi.org/10.4236/ojfd.2013.32009

[15] Kitchatinov, L.L., Rudiger, G. and Khomenko, G. (1994) Large-Scale Vortices in Rotating Stratified Disks. Astronomy \& Astrophysics, 287, 320.

[16] Moffat, H.K. (1978) Magnetic Field Generation in Electrically Conducting Fluids. Cambridge University Press, Cambridge.

[17] Gradshteyn, I.S. and Ryzhik, I.M. (2000) Table of Integrals, Series, and Prodacts. Academic Press, London. 